ISSN 0103-9954

\title{
ESTRUTURA POPULACIONAL E REGENERAÇÃO DE ESPÉCIES ARBÓREAS NA FLORESTA NACIONAL DE SÃO FRANCISCO DE PAULA, RIO GRANDE DO SUL
}

\author{
POPULATION STRUCTURE AND REGENERATION OF ARBOREAL SPECIES IN THE NATIONAL \\ FOREST OF SÃO FRANCISCO DE PAULA, RIO GRANDE DO SUL STATE
}

\author{
Angela Luciana de Avila ${ }^{1}$ Maristela Machado Araujo ${ }^{2}$ Solon Jonas Longhi ${ }^{3}$ Paulo Renato Schneider ${ }^{3}$ \\ João Olegário Pereira de Carvalho ${ }^{4}$
}

\section{RESUMO}

Este estudo teve como objetivo caracterizar a estrutura populacional e os aspectos da regeneração de Araucaria angustifolia (Bertol.) Kuntze, Blepharocalyx salicifolius (Kunth) O. Berg e Ilex paraguariensis A. St.-Hil em diferentes condições ambientais e histórico de perturbação na Floresta Nacional de São Francisco de Paula - RS. Foram utilizados seis conglomerados de um hectare, com 16 parcelas $(20$ × 20 m) cada, nos quais foram amostradas as espécies em três classes de tamanho. A classe de tamanho II (CT II - diâmetro a altura do peito $(\mathrm{DAP}) \geq 9,55 \mathrm{~cm})$ foi inventariada nas 96 parcelas, a CT I $(4,8 \leq$ DAP $<9,55 \mathrm{~cm})$ em 36 subparcelas de $10 \times 10 \mathrm{~m}$ e a regeneração natural estabelecida (RNE) $(1 \leq \mathrm{DAP}<4,8$ $\mathrm{cm}$ ) em 36 células de 3,16 × 3,16 m. Em todas as parcelas de $20 \times 20 \mathrm{~m}$ foram avaliados o banco de plântulas (BP - altura $\geq 30 \mathrm{~cm}$ e DAP $<1 \mathrm{~cm}$ ), a chuva de sementes (CS) e o banco de sementes do solo (BS). Os dados foram analisados considerando todos os ambientes conjunta e separadamente para os agrupamentos identificados em estudo prévio na área. Foram avaliadas a estrutura diamétrica para a CT II e densidade e frequência absoluta para todas as etapas do ciclo de vida (CS, BS, BP, RNE, CT I e CT II). Os resultados indicaram grande variabilidade na densidade destas espécies nos diferentes ciclos de vida e ambientes florestais. Araucaria angustifolia demonstrou falhas no processo de regeneração em ambiente com menor histórico de perturbação, não apresentando padrão de distribuição "J invertido" (CT II) e menor capacidade de regeneração em relação às demais espécies nos mecanismos de regeneração (CS, BS e BP). Ilex paraguariensis e Blepharocalyx salicifolius apresentaram melhor estabilidade populacional em ambiente com menor nível de interferência antrópica (CT II). A primeira espécie apresentou melhores condições de regeneração em ambientes de solo com boa drenagem e a segunda espécie demonstrou preferência para solo mais úmido. Além disso, falhas no processo de regeneração das populações foram detectadas e variaram de acordo com as condições ambientais e o histórico de perturbação de cada área. Assim, identifica-se a importância de considerar os diferentes ambientes florestais, a autoecologia das espécies e de aplicar possíveis intervenções que visem melhorar a regeneração e equilibrar a dinâmica populacional destas espécies.

Palavras-chave: Araucaria angustifólia; Blepharocalyx salicifolius; Floresta Ombrófila Mista; distribuição de frequência.

1 Engenheira Florestal, Msc., Doutoranda no Instituto de Silvicultura, Faculdade de Recursos Ambientais e Naturais, Universidade de Freiburg, Tennenbacherstr. 4, 79085, Freiburg im Breisgau, Alemanha. angeladeavila@gmail.com

2 Engenheira Florestal, Dr ${ }^{\mathrm{a}}$., Professora Adjunta do Departamento de Ciências Florestais, Centro de Ciências Rurais, Universidade Federal de Santa Maria, Av. Roraima, 1000, CEP 97105-900, Santa Maria (RS), Brasil. araujo.maristela@gmail.com

3 Engenheiro Florestal, Dr., Professor Adjunto do Departamento de Ciências Florestais, Centro de Ciências Rurais, Universidade Federal de Santa Maria, Av. Roraima, 1000, CEP 97105-900, Santa Maria (RS), Brasil. longhi.solon@gmail.com.paulors@smail.ufsm.br

4 Engenheiro Florestal, PhD., Professor do Mestrado em Ciências Florestais, Universidade Federal Rural da Amazônia, Av. Presidente Tancredo Neves, 2501, Caixa Postal, 917, CEP 66077-530, Belém (PA), Brasil. olegario.carvalho@gmail.com

Recebido para publicação em 4/10/2010 e aceito em 18/11/2014

Ci. Fl., v. 26, n. 3, jul.-set., 2016 


\begin{abstract}
The aim of this study was to characterize the population structure and aspects of regeneration of Araucaria angustifolia (Bertol.) Kuntze, Blepharocalyx salicifolius (Kunth) O. Berg and Ilex paraguariensis A. St.Hil. in different environmental and disturbance conditions in the National Forest of São Francisco de Paula, RS state. Six conglomerates of one hectare were used, with 16 plots $(20 \times 20 \mathrm{~m})$ each, where the species were sampled in three size classes. The size class II (SC II - diameter at breast height (DBH) $\geq 9.55 \mathrm{~cm}$ ) was sampled in 96 plots, the SC I $(4.8 \leq \mathrm{DBH}<9.55 \mathrm{~cm})$ in 36 subplots of $10 \times 10 \mathrm{~m}$, and the established natural regeneration $(\mathrm{ENR})(1 \leq \mathrm{DBH}<4.8 \mathrm{~cm})$ in 36 cells of $3.16 \times 3.16 \mathrm{~m}$. The seedling bank $(\mathrm{SB}-$ height $\geq 30$ $\mathrm{cm}$ and $\mathrm{DBH}<1 \mathrm{~cm}$ ), the seed rain (SR) and the soil seed bank (SSB) were sampled in all plots of $20 \times 20$ $\mathrm{m}$. The data were analysed by taking into account all the different vegetation groups together and separately for each cluster identified in a previous study. For SC II, the frequency distribution of DBH was analysed combined with the analysis of absolute density and frequency for all life stages (SR, SSB, SB, ENR, SC I and SC II). The results indicated high variability in density of these species among the different life stages and environmental conditions. Araucaria angustifolia presented gaps in the regeneration process in areas that had low past disturbance, not showing the "reverse-J" distribution (SC II) and with lower regeneration capacity in relation to the other species (SR, SSB and SB). Ilex paraguariensis and Blepharocalyx salicifolius showed higher population stability in environment with low level of human interference (SC II). The first species demonstrated preference for soils with good drainage, while the second species for humid soils. Moreover, gaps in the regeneration process of different populations were detected, which changed according to environmental conditions and disturbance levels. Therefore, the results suggest the importance of taking into account the different vegetation groups, the species autecology and to apply possible interventions that aim to improve regeneration and balance the population dynamics of these species.
\end{abstract}

Keywords: Araucaria angustifolia; Blepharocalyx salicifolius; mixed rain forest; frequency distribution.

\section{INTRODUÇÃO}

Os efeitos procedentes das ações antrópicas causam alterações na dinâmica das florestas nativas, influenciando a estrutura e a regeneração das populações de espécies florestais. No Rio Grande do Sul, a maior parte da vegetação florestal passou por algum nível de interferência, restando poucas áreas primárias. Assim, as Unidades de Conservação, comumente, contêm um mosaico de estádios de sucessão que resguardam e protegem amostras dos ecossistemas nativos e constituem laboratórios naturais para estudos ecológicos.

Neste contexto, a aplicação de práticas embasadas no conhecimento sobre o processo de sucessão pode favorecer a restauração de ecossistemas e a conservação de espécies ameaçadas (BEGON et al., 2007). Após a incidência de um distúrbio, a sucessão secundária se constitui numa sequência de alterações na composição florística e na estrutura da comunidade (CARVALHO, 1997). O sucesso na dispersão de sementes, posterior germinação e estabelecimento de plântulas determina a composição florística da floresta em sucessão (NORDEN et al., 2009). Além disso, a presença de indivíduos regenerantes é um fator decisivo para a consequente estabilidade populacional das espécies dominantes em fases subsequentes (HARTSHORN, 1980).

O estudo da distribuição dos indivíduos em diferentes classes de tamanho possibilita identificar a influência do histórico de perturbação da área sobre a dinâmica das populações (RICHARDS, 1998), permitindo verificar se as espécies estão em equilíbrio com o estádio sucessional da floresta (JARENKOW, 1985). Costa Júnior et al. (2008) relataram a importância de se conduzir, de forma conjunta, estudos sobre a regeneração e a estrutura populacional das espécies, visando a uma análise e inferências mais seguras sobre o comportamento da distribuição diamétrica. Adicionalmente, deve-se considerar que a vegetação não se distribui de forma homogênea (MATTEUCCI e COLMA, 1982), sendo necessário conhecer a dinâmica das espécies em diferentes condições ambientais.

Considerando que a atual política ambiental 
definiu as Florestas Nacionais como áreas passíveis de concessão para a prática de manejo florestal sustentável, através da Lei Federal ${ }^{\circ} 11.284$, de 2 de março de 2006 (BRASIL, 2006), é necessário o entendimento sobre a estrutura populacional e a ecologia da regeneração das espécies, para dar suporte à tomada de decisões. Segundo Sabogal (1992), o manejo das florestas naturais pode ser realizado com vistas a sua proteção, regeneração e restauração, bem como visando ao uso sustentável dos recursos florestais, sendo necessário o conhecimento sobre as bases ecológicas que regem estes ecossistemas. Assim, o entendimento sobre a dinâmica das espécies e seus respectivos papéis no processo de renovação da floresta é de fundamental importância (BAZZAZ, 1991).

No que se refere aos estudos na Floresta Ombrófila Mista do sul do Brasil que buscaram avaliar aspectos da estrutura populacional de espécies arbóreas, podem ser citados Caldato et al. (1999), Canalez et al. (2006) e Nascimento et al. (2001). No que diz respeito aos mecanismos de regeneração pode-se citar Caldato et al. (1996) e Chami et al. (2011). Entretanto, não foram constatadas análises de populações, contemplando desde a dispersão das sementes até os indivíduos adultos.

Araucaria angustifolia (Bertol.) Kuntze (pinheiro-brasileiro), Blepharocalyx salicifolius (Kunth) O. Berg (murta) e Ilex paraguariensis A. St.-Hil. (erva-mate) são espécies-chaves e características da Floresta Ombrófila Mista. A primeira, segundo Longhi (1980), é uma espécie emergente e de maior expressão fisionômica e dominância nesta fitofisionomia. A segunda espécie pertence à família Myrtaceae, a qual apresenta grande densidade e dispersão tanto neste tipo florestal como em todo o estado do Rio Grande do Sul. A terceira espécie ocorre frequentemente em associação com o pinheiro-brasileiro. Estas espécies apresentam interesse econômico, medicinal e alimentar para o homem, assim como importância ecológica para a recuperação de ecossistemas degradados, servindo também de alimento para a fauna. Porém, o extrativismo não planejado, principalmente, no caso do pinheiro-brasileiro e da erva-mate, provocou fortes alterações na composição e estrutura da Floresta Ombrófila Mista (RIO GRANDE DO SUL, 2002).

Desta forma, este estudo buscou caracterizar a estrutura populacional e a regeneração de Araucaria angustifolia, Blepharocalyx salicifolius e Ilex paraguariensis, em diferentes condições ambientais e histórico de perturbação na Floresta Nacional de São Francisco de Paula - RS, Brasil, com o objetivo de fornecer informações que podem ser usadas para o desenvolvimento de técnicas que possibilitem, por exemplo, a conservação destas espécies, o uso sustentável e a recuperação ambiental de ecossistemas da Floresta Ombrófila Mista.

\section{MATERIAL E MÉTODO}

Área de estudo

A pesquisa foi desenvolvida na Floresta Nacional de São Francisco de Paula (FLONASFP / $29^{\circ} 24^{\prime}$ e $29^{\circ} 27^{\prime} \mathrm{S}$; $50^{\circ} 22^{\prime}$ e $50^{\circ} 25^{\prime} \mathrm{W}$ ), em São Francisco de Paula - RS, Brasil. A área total corresponde a 1.606,6 ha e, destes, aproximadamente, $56 \%$ são ocupados por floresta nativa (BRASIL, 2000). O relevo pertence à Unidade Planalto das Araucárias (HERRMANN e ROSA, 1990) e a altitude máxima da FLONA corresponde a $930 \mathrm{~m}$ (BRASIL, 2000). Segundo o Sistema Brasileiro de Classificação de Solos (SBCS), a região apresenta Cambissolo Húmico Alumínico Típico - CHa 1 (EMBRAPA, 1999), o qual é considerado ácido e apresenta valores elevados de alumínio trocável (MOSER, 1990). O clima da área de estudo, conforme classificação de Köppen, é do tipo "Cfb" (mesotérmico e superúmido), com chuvas distribuídas ao longo do ano e temperatura média anual de $14,5^{\circ} \mathrm{C}$ (MALUF, 2000). A região apresenta pluviosidade anual de aproximadamente $2.500 \mathrm{~mm}$ (NIMER, 1990).

A vegetação característica é constituída pela Floresta Ombrófila Mista (FOM) que apresenta, em geral, dossel multiestratificado com diferentes padrões fisionômicos e estruturais, sendo que, logo abaixo da copa da Araucaria angustifolia, é encontrado um estrato contínuo de espécies latifoliadas (LEITE, 2002). Este é composto, de 60 a $70 \%$, por espécies de Lauraceae, Aquifoliaceae e Sapindaceae. O estrato inferior é formado predominantemente por espécies de Myrtaceae (QUADROS e PILLAR, 2002). Araujo et al. (2010), em pesquisa realizada na FLONA-SFP e utilizando a estrutura amostral do Projeto PELD/CNPq (Projeto Ecológico de Longa Duração) Site 9, identificaram que Araucaria angustifolia, Blepharocalyx salicifolius e Ilex paraguariensis encontram-se entre as espécies com maior valor de importância na floresta. Além disso, foram observados três 
agrupamentos na vegetação conforme a condição ambiental e o histórico de perturbação (Tabela 1). Esses grupos foram utilizados como base para a análise da estrutura populacional das espécies no presente estudo.

\section{Amostragem dos dados}

A pesquisa utilizou a estrutura amostral do Projeto PELD/CNPqSite9-intitulado "Conservação e Manejo Sustentável de Ecossistemas Florestais Bioma Floresta de Araucária e suas Transições". Nesse projeto foram instalados seis conglomerados de 1 ha cada, de maneira a amostrar as diferentes variações ambientais e os estádios sucessionais existentes na área de estudo. As amostragens das populações de Araucaria angustifolia, Blepharocalyx salicifolius e Ilex paraguariensis foram realizadas nos conglomerados, desconsiderando $10 \mathrm{~m}$ de bordadura. Cada conglomerado é composto de 16 parcelas de 20 x $20 \mathrm{~m}$. A vegetação correspondente à classe de tamanho II (CT II - DAP $\geq 9,55 \mathrm{~cm}$ ) foi amostrada em 96 parcelas de 20 × $20 \mathrm{~m}$ (3,84 ha). Para as avaliações da CT I e regeneração natural estabelecida (RNE) foram sorteadas seis parcelas em cada conglomerado, e dentro de cada uma destas parcelas uma subparcela de $10 \times 10 \mathrm{~m}$ foi sorteada. No total, a classe de tamanho I (CT I - 4,8 $\leq$ DAP $<$ 9,55 m) foi amostrada em 36 subparcelas de $10 \times 10$ $\mathrm{m}(0,36 \mathrm{ha})$ e, no vértice sudoeste destas, em 36 pequenas subparcelas de 3,16 × 3,16 m (0,04 ha) foi inventariada a RNE. Foi mensurado o DAP em todas as classes de tamanho.

O centro de cada parcela de $20 \times 20 \mathrm{~m}$ foi utilizado como referência para a amostragem dos mecanismos de regeneração natural: chuva de sementes (CS), banco de sementes do solo (BS) e banco de plântulas (BP). A CS foi avaliada em 16 coletores circulares $\left(1 \mathrm{~m}^{2}\right.$ cada $)$ por conglomerado (total de 96 coletores). As coletas foram mensais, de fevereiro de 2008 a janeiro de 2009. O material foi armazenado em sacos plásticos identificados e conduzido ao Laboratório de Silvicultura (Universidade Federal de Santa Maria - UFSM) para identificação e quantificação das sementes aparentemente viáveis e com tamanho igual ou maior que $1 \mathrm{~mm}$ (ARAUJO et al. 2004).

O BS foi amostrado a $3 \mathrm{~m}$ dos coletores da CS, em sentido aleatório, através da utilização de gabarito de ferro $(0,25 \times 0,25 \mathrm{~m})$, coletando-se os primeiros $5 \mathrm{~cm}$ do solo, desconsiderando a camada de serapilheira (ARAUJO et al., 2004). A coleta foi realizada em outubro de 2008 e o material foi conduzido à casa de vegetação do Laboratório de Silvicultura (UFSM), com o objetivo de estimular a germinação. Cada amostra foi distribuída no interior de uma bandeja com vermiculita, sendo estas dispostas de forma aleatória (sorteio), sobre bancadas cobertas com tela de náilon $(50 \%)$ para evitar a contaminação de propágulos externos. A verificação de possíveis contaminantes foi realizada pelo uso de quatro bandejas-testemunhas, contendo apenas vermiculita (ARAUJO et al. 2004). A identificação e contagem das plântulas germinadas foram realizadas mensalmente, até o $210^{\circ}$ dia após a coleta.

O BP (altura $\geq 30 \mathrm{~cm}$ e DAP $<1 \mathrm{~cm}$ ) foi amostrado em março de 2009, em 96 subunidades de $2 \times 2 \mathrm{~m}$ (0,04 ha), localizadas em sentido aleatório e distante $4 \mathrm{~m}$ do coletor da CS, sendo realizada a contagem do número de indivíduos amostrados por espécie. A aleatoriedade para o BS e BP foi determinada por meio de sorteio realizado previamente, considerando as direções norte, sul, leste e oeste.

\section{Análise dos dados}

A distribuição de frequências e a estrutura horizontal foram estudadas considerando todos os ambientes conjuntamente e separadamente para os agrupamentos detectados por Araujo et al. (2010) (Tabela 1) e corroborados em estudo realizado por Avila et al. (2011) sobre a classificação da regeneração na área de estudo. Desta forma, foi possível observar os padrões ao se considerar a área como homogênea ou ao tomar em consideração as diferenças ambientais e o histórico de perturbação nos diferentes agrupamentos.

A CT II das populações foi avaliada por meio da distribuição de frequências em classes de diâmetro. $\mathrm{O}$ número de classes para distribuição diamétrica foi determinado pela fórmula de Sturges (ver FINGER, 1992). Assim, para Araucaria angustifolia e Blepharocalyx salicifolius, foram delimitadas nove classes com a amplitude de 10 cm $(9,5-19,49 \mathrm{~cm} ; 19,5-29,49 \mathrm{~cm} ; . . ; 89,5-99,49$ $\mathrm{cm})$, enquanto que para Ilex paraguariensis, foram delimitadas 9 classes com amplitude de $5 \mathrm{~cm}(9,5-$ $14,49 \mathrm{~cm} ; \ldots ; 54,5-59,49 \mathrm{~cm})$.

A distribuição de frequências foi avaliada através da análise de regressão, utilizando o centro de classe como variável independente e o 
TABELA 1: Características florísticas, estruturais e histórico de interferência antrópica em cada agrupamento identificado em remanescente da Floresta Ombrófila Mista na Floresta Nacional de São Francisco de Paula, São Francisco de Paula - RS, Brasil.

TABLE 1: Floristic characteristics, structure and historic of anthropogenic interference in each cluster identified in a Mixed Rain Forest remnant in the National Forest of São Francisco de Paula, São Francisco de Paula, RS state, Brazil.

\begin{tabular}{|c|c|c|c|}
\hline $\begin{array}{c}\text { Grupo } \\
\text { (área abrangida) }\end{array}$ & Caracterização florística ${ }^{1}$ & $\begin{array}{l}\text { Espécies com maior } \\
\text { VI }(\%)^{2} \text { em ordem } \\
\text { decrescente }^{1}\end{array}$ & $\begin{array}{l}\text { Histórico de } \\
\text { interferência }^{3}\end{array}$ \\
\hline $\begin{array}{l}\text { Grupo 1: } \\
\text { Ambiente de encosta } \\
(0,76 \text { ha })\end{array}$ & $\begin{array}{l}55 \text { espécies } \\
42 \text { gêneros } \\
23 \text { famílias } \\
877 \text { indivíduos } \text { ha }^{-1}\end{array}$ & $\begin{array}{l}\text { Siphoneugena reitzii } \\
\text { Ilex brevicuspis } \\
\text { Podocarpus lambertii } \\
\text { Araucaria angustifolia }\end{array}$ & $\begin{array}{l}\text { 1940/50/70: exploração da } \\
\text { Araucaria angustifolia e outras } \\
\text { espécies; área incorporada } \\
\text { à FLONA-SFP em } 1987 \text {; } \\
\text { 1990: eliminação dos fatores } \\
\text { interferentes. }\end{array}$ \\
\hline $\begin{array}{l}\text { Grupo } 2 \text { : } \\
\text { Ambiente com } \\
\text { declividade média a } \\
\text { acentuada }(1,72 \text { ha })\end{array}$ & $\begin{array}{l}66 \text { espécies } \\
51 \text { gêneros } \\
30 \text { famílias } \\
809 \text { indivíduos } \text { ha }^{-1}\end{array}$ & $\begin{array}{l}\text { Araucaria angustifolia } \\
\text { Casearia decandra } \\
\text { Blepharocalyx } \\
\text { salicifolius } \\
\text { Ilex paraguariensis }\end{array}$ & $\begin{array}{l}\text { Menor nível de interferência } \\
\text { antrópica, com registro de acesso } \\
\text { à área por animas (suínos) até, } \\
\text { aproximadamente, } 1945 \text {. }\end{array}$ \\
\hline $\begin{array}{l}\text { Grupo 3: } \\
\text { Ambiente plano e } \\
\text { úmido (1,36 ha) }\end{array}$ & $\begin{array}{l}53 \text { espécies } \\
38 \text { gêneros } \\
24 \text { famílias } \\
667 \text { indivíduos ha-1 }\end{array}$ & $\begin{array}{c}\text { Sebastiania } \\
\text { commersoniana } \\
\text { Blepharocalyx } \\
\text { salicifolius } \\
\text { Ilex brevicuspis } \\
\text { Araucaria angustifolia }\end{array}$ & $\begin{array}{l}\text { 1940/50: exploração da Araucaria } \\
\text { angustifolia; retirada de madeira } \\
\text { morta para fins energéticos; abrigo } \\
\text { para o gado e equinos. }\end{array}$ \\
\hline
\end{tabular}

Em que: ${ }^{1}$ Adaptado de Araujo et al. (2010); ${ }^{2}$ VI (\%): Valor de importância avaliado nas plantas com DAP $\geq 9,55 \mathrm{~cm}$; ${ }^{3}$ Soligo (com. pess.).

número de indivíduos em cada classe de diâmetro como variável dependente. Este procedimento foi realizado com o objetivo de obter o coeficiente angular (inclinação) das equações de regressão, utilizado como indicativo da estrutura da população (LYKKE, 1998; McLAREN, 2005; OBIRI et al., 2002; MWAVU e WITKOWSKI, 2009).

Ométodo de Box Cox foi empregado visando atender dois pressupostos da análise de regressão (homogeneidade das variâncias e normalidade dos resíduos) e identificar qual transformação $(\lambda)$ apresentou melhor adequação aos dados. No caso de $\lambda=0$, por definição $\mathrm{Y}^{\prime}=\log _{\mathrm{e}} \mathrm{Y}$, então a equação a ser ajustada é a função de Meyer: $\ln \mathrm{Ni}=\mathrm{b}_{0}+$ $\mathrm{b}_{1}{ }^{*} \mathrm{~d}$, onde $\mathrm{Ni}=$ número de indivíduos $\mathrm{ha}^{-1}$ e $\mathrm{d}=$ centro de classe de diâmetro. A independência dos resíduos não foi testada, considerando-se que a amostragem utilizada (aleatória) possivelmente atende a suposição dos resíduos independentes (SCHNEIDER et al., 2009).

Adicionalmente, foi realizada a análise da estrutura horizontal com o intuito de subsidiar informações para melhor compreensão da estrutura das populações nas diferentes etapas do ciclo de vida. Assim, a CS e o BS foram analisados por meio da densidade absoluta (DA) de sementes $\mathrm{m}^{-2}$ e frequência absoluta (FA) em porcentagem. Para o BP, RNE, CT I e CT II foram avaliados a DA de indivíduos ha ${ }^{-1}$ e a FA (\%).

\section{RESULTADOS E DISCUSSÃO}

Os máximos valores das classes de diâmetro observados para os indivíduos na área amostrada (3,84 ha) corresponderam ao centro de classe de 94,5 $\mathrm{cm}$ para Araucaria angustifolia e Blepharocalyx salicifolius e $52 \mathrm{~cm}$ para Ilex paraguariensis. $\mathrm{O}$ indivíduo com maior DAP amostrado foi um exemplar de murta com $111 \mathrm{~cm}$.

Araucaria angustifolia demonstrou distribuição de frequência descontínua, não apresentando o padrão "J invertido", quando 
considerado todos os ambientes florestais amostrados (3,84 ha). Este comportamento pode ser melhor compreendido ao se analisar e interpretar os padrões de distribuição de frequência observados nos diferentes ambientes florestais. No Grupo 1 (ambiente de encosta), a espécie apresentou elevada densidade de indivíduos nas classes de regeneração natural (BP, RNE, CT I) e baixa densidade de indivíduos com DAP $\geq 9,55 \mathrm{~cm}$ (Figura 1B). Estes resultados podem estar relacionados com o recente histórico de extrativismo registrado para esse ambiente (Tabela 1). A baixa densidade de indivíduos de maior diâmetro para a espécie, devido a efeitos passados de exploração, também foi relatada por Souza (2007) em estudo sobre a estrutura populacional da espécie em diferentes fragmentos da FOM no RS. Segundo Hess et al. (2000), a distribuição em classes de diâmetro é altamente influenciada pela exploração madeireira, sendo que, quanto mais intenso este processo maior o recrutamento de plântulas e mais pronunciada a redução no número de indivíduos com diâmetros potenciais para uso econômico.

O Grupo 2, ambiente com declividade média a acentuada e que sofreu menor nível de interferência antrópica, apresentou a maior densidade de indivíduos de Araucaria angustifolia na CT II (DAP $\geq 9,55 \mathrm{~cm}$ ), porém, com oscilações entre as classes de diâmetro (Figura 1C). Foram observadas falhas no processo de regeneração pela ausência e baixa densidade de indivíduos nas menores classes, para a RNE e CT I, respectivamente (Figura 1C). Carvalho (2003) relatou que a espécie, geralmente, apresenta problemas na regeneração natural em ambientes pouco perturbados, devido aos baixos índices de luminosidade no interior da floresta. Além disso, as afirmações de Souza et al. (2008) confirmam este comportamento, pois, segundo os autores, a Araucaria angustifolia é uma espécie pioneira de longa vida, que necessita de alterações na floresta, para que a regeneração ocorra com sucesso.

Segundo Schneider (com. pess.), os indivíduos de Araucaria angustifolia permanecem suprimidos sob o dossel da floresta à espera de condições adequadas para o desenvolvimento, principalmente, no que se refere à luminosidade, sendo comum a dificuldade na leitura dos anéis de crescimento na região próxima à medula em árvores adultas desta espécie devido às reduzidas taxas de crescimento na fase juvenil. Richards (1998) relatou que algumas espécies demandantes de luz apresentam habilidade para sobreviver com crescimento reduzido sob o dossel, porém, na presença de luz recrutam imediatamente para as classes posteriores. No caso do pinheiro-brasileiro, como espécie pioneira e heliófita, os seus indivíduos permanecem sob o dossel com caule extremamente fino e copa reduzida (REITZ et al., 1983) aguardando condições favoráveis.

Neste mesmo ambiente (Grupo 2), os resultados sugerem que a limitação no processo de recrutamento e desenvolvimento de indivíduos encontra-se nas menores classes de tamanho, principalmente na RNE. Possivelmente, o longo período em que os indivíduos permanecem à espera de condições para o seu desenvolvimento no sub-bosque da floresta (ex. BP), pode ser excessivo causando sua mortalidade e impedindo o seu recrutamento para classes posteriores. Além disso, o menor potencial do pinheiro-brasileiro, no processo de competição com algumas espécies de angiospermas, também reforça o padrão de exclusão de indivíduos observados na regeneração, pois, segundo Lamprecht (1990), as angiospermas apresentam maior adaptabilidade e competitividade em relação às coníferas.

O Grupo 3 (ambiente plano e úmido) apresentou comportamento de distribuição de frequências semelhante à população de Araucaria angustifolia no Grupo 1, porém, com ausência de indivíduos na RNE (Figura 1D). A proximidade no padrão de distribuição de frequências, entre esses dois grupos, deve-se ao histórico de perturbação das duas áreas, considerando que ambas tiveram extrativismo de madeira e maior nível de interferência antrópica. Entretanto, no terceiro agrupamento, a exploração e a consequente abertura de clareiras ocorreram há mais tempo e, após 1940, as intervenções neste ambiente estiveram relacionadas à remoção de madeira de árvores mortas e pastoreio de animais (Tabela 1). Estes dois últimos fatores não provocaram grandes aberturas no dossel da floresta, possibilitando que este fosse recomposto gradualmente, ao mesmo tempo em que a luminosidade foi diminuindo no interior da floresta, pelo maior grau de desenvolvimento na estratificação do dossel, o que pode explicar a ausência de indivíduos na RNE do Grupo 3. Por outro lado, devido à exploração ter ocorrido em período mais recente no Grupo 1 (Tabela 1), as clareiras abertas ainda influenciam de maneira mais pronunciada a dinâmica da vegetação, permitindo que a regeneração natural se estabeleça e recrute.

As populações de Araucaria angustifolia 
apresentaram um pico de frequência no centro de classe, equivalente a $54,5 \mathrm{~cm}$ em todos os ambientes (Figura 1). Esta característica reflete, possivelmente, os efeitos de interferências decorridas na área, representando os indivíduos-testemunhas deste processo e que deram continuidade ao ciclo de vida da espécie na comunidade, principalmente, nos grupos 1 e 3. Neste contexto, Zanon (2007) avaliou o crescimento do pinheiro-brasileiro em plantios na FLONA-SFP com o uso de fitas dendrométricas na altura do DAP e encontrou um incremento corrente médio anual de $2,7 \mathrm{~mm}$, para árvores com idade de 40 anos. Considerando que na década de 1950, os indivíduos remanescentes da exploração do pinheirobrasileiro apresentavam cerca de $40 \mathrm{~cm}$ de DAP, o incremento acima descrito sugere que essas árvores deveriam apresentar atualmente aproximadamente $56,2 \mathrm{~cm}$, indicando a possibilidade dos mesmos serem testemunhas da exploração incidente no passado.

A transformação Box Cox (CT II) indicou valor de lambda igual à zero, como recomendado ou presente no intervalo de confiança, para todos os ambientes e espécies, sendo eficiente para atender aos pressupostos da análise de regressão. Araucaria angustifolia apresentou distribuição de frequência descontínua na floresta, o que refletiu na análise de regressão aplicada para identificar o valor de inclinação $\left(b_{1}\right)$ representativo da estrutura das populações, com valores muito baixos para o coeficiente de determinação $\left(\mathrm{R}^{2}\right)$, indicando que a referida espécie não apresentou ajuste dos dados para a função de Meyer, comumente utilizada, por explicar e ajustar a distribuição diamétrica em florestas naturais (PRODAM, 1968 apud SILVA et al., 2004).

Em todos os ambientes florestais (grupos), a Araucaria angustifolia está presente na chuva de sementes, porém, ausente no BS (Tabela 2), o que ocorre devido à característica recalcitrante de suas sementes (CARVALHO, 2003). Neste sentido, a presença de diásporos na área é uma característica potencial, que pode ser utilizada para o favorecimento na regeneração da espécie, enfatizando ainda,
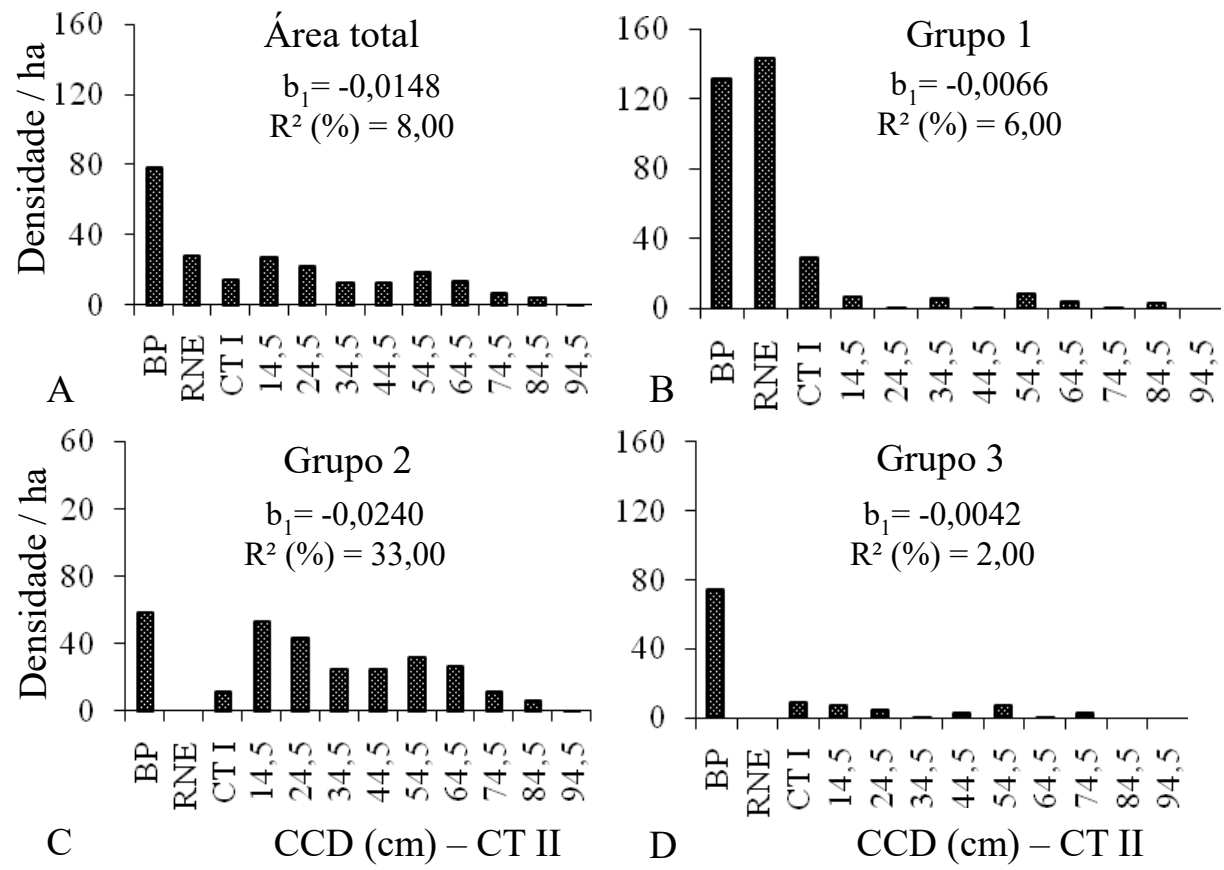

FIGURA 1: Distribuição de frequência para os indivíduos de Araucaria angustifolia em toda a área amostrada (A) e nos Grupos 1 (B), 2 (C) e 3 (D). Os valores referentes à inclinação (b $b_{1}$ ) e coeficiente de determinação $\left(\mathrm{R}^{2}\right)$ referem-se ao ajuste de equações para os indivíduos com $\mathrm{DAP} \geq 9,55 \mathrm{~cm}$ (CT II). Centro de classe de diâmetro (CCD).

FIGURE 1: Frequency distribution for individuals of Araucaria angustifolia in all sampled area (A) and Groups 1 (B), 2 (C) and 3 (D). The values for the slope $\left(b_{1}\right)$ and coefficient of determination $\left(\mathrm{R}^{2}\right)$ were obtained from adjustment of equations for individuals with $\mathrm{DBH} \geq 9.55 \mathrm{~cm}$ (CT II). Center of diameter class (CCD). 
a presença de indivíduos reprodutivos (CT II), bem distribuídos na área (Tabela 2). A frequência observada no BP, RNE e CT I indica forte agregação nestas classes de tamanho. Souza (2007) encontrou resultados semelhantes para indivíduos com tamanho correspondente, em diferentes fragmentos florestais no Rio Grande do Sul.

O Grupo 2 apresentou maior densidade na CS e na CT II, entretanto, a regeneração foi pouco pronunciada neste ambiente (Tabela 2). A alta produção de sementes pode ser justificada pela elevada densidade de indivíduos adultos registrados. Por outro lado, a deficiente regeneração natural da espécie pode estar relacionada à sua alta demanda por luz para regeneração e desenvolvimento (SOUZA, 2007; SOUZA et al., 2008; LONGHI et al., 2010). A maior densidade de indivíduos regenerantes do pinheiro-brasileiro foi observada no Grupo 1, ao mesmo tempo, que a CS e a densidade na CT II, apresentam-se muito menores, o que pode estar associado ao reduzido número de indivíduos reprodutivos neste agrupamento.

Blepharocalyx salicifolius apresentou uma distribuição diamétrica mais equilibrada em toda a área de amostragem ( $3,84 \mathrm{ha})$, quando comparada à Araucaria angustifolia, demonstrando padrão

TABELA 2: Valores de densidade e frequência absoluta para três espécies arbóreas, nas diferentes etapas do ciclo de vida, considerando os ambientes florestais da Floresta Nacional de São Francisco de Paula - RS, Brasil.

TABLE 2: Density and absolute frequency for three tree species (A, B and C) in different life cycle in vegetation clusters in the National Forest of São Francisco de Paula, RS, Brazil.

\begin{tabular}{|c|c|c|c|c|c|c|c|c|c|c|c|c|}
\hline \multicolumn{13}{|c|}{ Todos os ambientes florestais amostrados } \\
\hline \multirow{2}{*}{ Espécies $^{1}$} & \multicolumn{2}{|c|}{$\mathrm{CS}^{2}$} & \multicolumn{2}{|c|}{$\mathrm{BS}$} & \multicolumn{2}{|c|}{$\mathrm{BP}$} & \multicolumn{2}{|c|}{ RNE } & \multicolumn{2}{|c|}{ CT I } & \multicolumn{2}{|c|}{ CT II } \\
\hline & $\mathrm{DA}^{3}$ & $\mathrm{FA}^{4}$ & $\mathrm{DA}^{3}$ & FA & $\mathrm{DA}^{5}$ & FA & $\mathrm{DA}^{5}$ & FA & $\mathrm{DA}^{5}$ & FA & $\mathrm{DA}^{5}$ & FA \\
\hline A & 2,36 & 29,17 & 0,00 & 0,00 & 78,13 & 3,13 & 27,82 & 2,78 & 13,89 & 13,89 & 114,84 & 75,00 \\
\hline B & 288,21 & 46,88 & 0,00 & 0,00 & 468,75 & 1,04 & 111,27 & 11,11 & 19,44 & 19,44 & 48,18 & 80,21 \\
\hline $\mathrm{C}$ & 122,35 & 79,17 & 20,17 & 27,08 & 234,38 & 9,38 & 194,73 & 19,44 & 36,11 & 36,11 & 31,51 & 62,50 \\
\hline \multicolumn{13}{|c|}{ Ambiente de encosta (Grupo 1) } \\
\hline \multirow{2}{*}{ Espécies } & \multicolumn{2}{|c|}{$\mathrm{CS}$} & \multicolumn{2}{|c|}{ BS } & \multicolumn{2}{|c|}{ BP } & \multicolumn{2}{|c|}{ RNE } & \multicolumn{2}{|c|}{ CT I } & \multicolumn{2}{|c|}{ CT II } \\
\hline & $\mathrm{DA}^{3}$ & FA & $\mathrm{DA}^{3}$ & FA & $\mathrm{DA}^{5}$ & FA & $\mathrm{DA}^{5}$ & FA & $\mathrm{DA}^{5}$ & FA & $\mathrm{DA}^{5}$ & FA \\
\hline A & 0,53 & 10,53 & 0,00 & 0,00 & 131,58 & 5,26 & 143,08 & 14,29 & 28,57 & 28,57 & 30,26 & 57,89 \\
\hline B & 12,53 & 31,58 & 0,00 & 0,00 & 394,74 & 15,79 & 0,00 & 0,00 & 0,00 & 0,00 & 34,21 & 78,95 \\
\hline $\mathrm{C}$ & 299,37 & 89,47 & 54,62 & 21,05 & 131,58 & 5,26 & 0,00 & 0,00 & 0,00 & 0,00 & 30,26 & 63,16 \\
\hline \multicolumn{13}{|c|}{ Ambiente com declividade média a acentuada (Grupo 2) } \\
\hline \multirow{2}{*}{ Espécies } & \multicolumn{2}{|c|}{$\mathrm{CS}$} & \multicolumn{2}{|c|}{$\mathrm{BS}$} & \multicolumn{2}{|c|}{$\mathrm{BP}$} & \multicolumn{2}{|c|}{ RNE } & \multicolumn{2}{|c|}{ CT I } & \multicolumn{2}{|c|}{ CT II } \\
\hline & $\mathrm{DA}^{3}$ & FA & $\mathrm{DA}^{3}$ & FA & $\mathrm{DA}^{5}$ & FA & $\mathrm{DA}^{5}$ & FA & $\mathrm{DA}^{5}$ & FA & $\mathrm{DA}^{5}$ & FA \\
\hline A & 5,02 & 58,14 & 0,00 & 0,00 & 58,14 & 2,33 & 0,00 & 0,00 & 11,76 & 11,76 & 223,26 & 100,00 \\
\hline B & 0,65 & 20,93 & 0,00 & 0,00 & 581,40 & 23,26 & 58,91 & 5,88 & 41,18 & 41,18 & 49,42 & 76,74 \\
\hline $\mathrm{C}$ & 127,86 & 86,05 & 12,64 & 37,21 & 116,28 & 4,65 & 412,37 & 41,18 & 64,71 & 64,71 & 48,26 & 79,07 \\
\hline \multicolumn{13}{|c|}{ Ambiente úmido (Grupo 3) } \\
\hline \multirow{2}{*}{ Espécies } & \multicolumn{2}{|c|}{$\mathrm{CS}$} & \multicolumn{2}{|c|}{ BS } & \multicolumn{2}{|c|}{$\mathrm{BP}$} & \multicolumn{2}{|c|}{ RNE } & \multicolumn{2}{|c|}{ CT I } & & \\
\hline & $\mathrm{DA}^{3}$ & FA & $\mathrm{DA}^{3}$ & FA & $\mathrm{DA}^{5}$ & FA & $\mathrm{DA}^{5}$ & FA & $\mathrm{DA}^{5}$ & FA & $\mathrm{DA}^{5}$ & FA \\
\hline A & 0,03 & 2,94 & 0,00 & 0,00 & 73,53 & 2,94 & 0,00 & 0,00 & 8,33 & 8,33 & 25,00 & 52,94 \\
\hline B & 805,94 & 88,24 & 0,00 & 0,00 & 367,65 & 14,71 & 250,35 & 25,00 & 0,00 & 0,00 & 54,41 & 85,29 \\
\hline $\mathrm{C}$ & 16,47 & 52,94 & 10,38 & 0,18 & 441,18 & 17,65 & 0,00 & 0,00 & 16,67 & 16,67 & 11,03 & 41,18 \\
\hline
\end{tabular}

Em que: ${ }^{1}$ A: Araucaria angustifolia, B: Blepharocalyx salicifolius, $C$ : Ilex paraguariensis; ${ }^{2} \mathrm{CS}$ : Chuva de sementes; BS: Banco de sementes do solo; BP: Banco de plântulas; RNE: Regeneração natural estabelecida; CT I: Classe de tamanho I; CT II: Classe de tamanho II; ${ }^{3} \mathrm{DA}$ : Densidade absoluta $\mathrm{m}^{-2}$; ${ }^{4} \mathrm{FA}$ : Frequência absoluta (\%); ${ }^{5} \mathrm{DA}$ : Densidade absoluta ha-1. 
próximo do "J invertido" (Figura 2A). Contudo, ao se analisarem as populações nos diferentes ambientes, identificou-se que nos Grupos 1 e 3 (Figuras 2B e 2D) os indivíduos pertencentes à CT II apresentaram a distribuição diamétrica decrescente e nas menores classes ocorreram falhas no processo de regeneração, com ausência de indivíduos na RNE e CT I para Grupo 1 e, na CT I, para o Grupo 3. Este comportamento pode demonstrar instabilidade populacional, pois, segundo Jarenkow (1985) e Martins (1993), a ausência de indivíduos em alguma categoria do ciclo de vida pode comprometer sua permanência na comunidade. Nesse sentido, pode-se inferir que as mudanças ambientais referentes ao histórico de perturbação influenciaram negativamente o desenvolvimento de Blepharocalyx salicifolius, considerando-se que a mesma não pertence ao grupo ecológico de pioneiras (CARVALHO, 2006), remetendo à necessidade de menores intensidades de luminosidade nas fases inicias de germinação e estabelecimento. Dessa forma, os indivíduos já estabelecidos na ocasião das perturbações, permaneceram na comunidade e, no presente estádio sucessional, atuam como fonte de sementes para a retomada do equilíbrio no ciclo regenerativo da espécie.

O Grupo 2 apresentou elevada proporção de indivíduos no banco de plântulas (Figura 2C), decaindo fortemente na RNE, sendo que, o número de indivíduos, não apresentou grandes variações, a partir desta classe de tamanho. $\mathrm{O}$ ajuste de equações indicou valores de inclinação negativos, para as populações de Blepharocalyx salicifolius, tanto na amostragem que considerou todos os ambientes (3,84 ha) como nos diferentes ambientes florestais (Figura 2). Para essa espécie, a função de Meyer apresentou os melhores ajustes. $\mathrm{O}$ menor valor de inclinação $\left(b_{1}\right)$ e o melhor ajuste $\left(R^{2}\right)$ foram encontrados no Grupo 2, no qual a distribuição de frequências sugere que a população encontra-se em melhor equilíbrio.

A murta apresentou elevados valores de densidade na chuva de sementes para o Grupo 3, no qual também foram observadas as maiores densidades absolutas para os indivíduos da CT II (Tabela 2). O BS não apresentou indivíduos de murta, indicando que a mesma não apresenta este mecanismo como estratégia de regeneração, devido ao comportamento recalcitrante de suas sementes (CARVALHO, 2006). No BP, observou-se que a

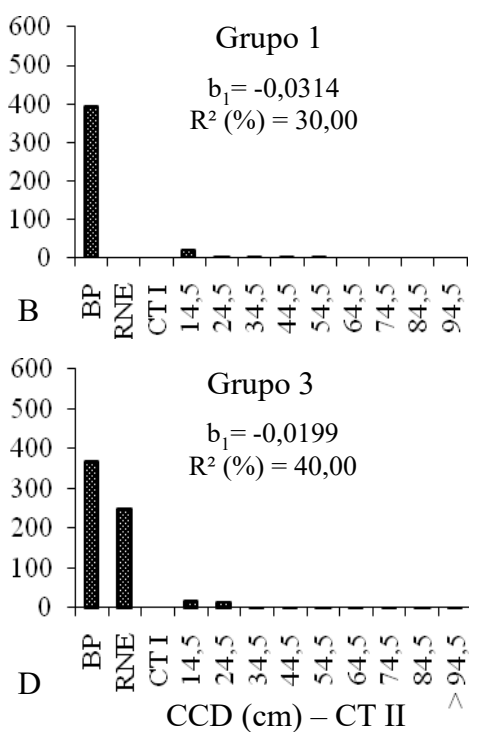

FIGURA 2: Distribuição de frequência para os indivíduos de Blepharocalyx salicifolius em toda a área amostrada (A) e nos Grupos 1 (B), 2 (C) e 3 (D). Os valores referentes à inclinação $\left(b_{1}\right)$ e coeficiente de determinação $\left(\mathrm{R}^{2}\right)$ referem-se ao ajuste de equações para os indivíduos com DAP $\geq 9,55 \mathrm{~cm}$ (CT II). Centro de classe de diâmetro (CCD).

FIGURE 2: Frequency distribution for individuals of Blepharocalyx salicifolius in all sampled area (A) and Groups 1 (B), 2 (C) and 3 (D). The values for the slope $\left(b_{1}\right)$ and coefficient of determination $\left(\mathrm{R}^{2}\right)$ were obtained from adjustment of equations for individuals with $\mathrm{DBH} \geq 9.55 \mathrm{~cm}$ (CT II). Center of diameter class (CCD). 
espécie encontra-se bem representada em todos os ambientes, com maior densidade no Grupo 2, no qual foi amostrada em todas as classes de tamanho da vegetação. Contudo, observou-se a ausência na CT I, nos Grupos 1 e 3, existindo possivelmente algum fator que limita o recrutamento dos indivíduos nesta classe e desta para a população adulta (Tabela 2).

A distribuição das frequências diamétricas para Ilex paraguariensis considerando toda a área amostrada (3,84 ha) sugere que a população encontra-se com boa capacidade regenerativa com elevado número de indivíduos nas menores classes, decrescendo com o aumento na dimensão do diâmetro (Figura 3A). No entanto, para os Grupos 1 e 3, assim como para Blepharocalyx salicifolius, foram encontradas falhas no processo de regeneração com ausência de indivíduos em ao menos uma das classes correspondentes a RNE e CT I (Figuras 3B e 3D), apresentando ainda oscilações na distribuição de frequências para a CT II. No Grupo 2, a regeneração de Ilex paraguariensis demonstrou maior equilíbrio, porém, o banco de plântulas apresentou menor densidade que a classe imediatamente posterior (Figura 3C).

A análise da distribuição, pelo ajuste de equações para a CT II, indicou valores negativos de inclinação para todas as populações e o melhor ajuste da função de Meyer foi obtido para o Grupo 2 , assim como, o menor valor de $b_{1}$, indicando que esta população apresenta-se autorregenerativa.

A análise da estrutura horizontal nas classes de tamanho demonstrou que a espécie apresenta-se bem distribuída em todas as etapas do ciclo de vida, quando se considera toda a área amostrada e o Grupo 2 (Tabela 2). A alta densidade observada no banco de sementes decorre de sua dormência morfológica, pela imaturidade do embrião, característica da família Aquifoliacae (BORGHETTI, 2004). Assim, as mesmas permanecem no solo completando a maturação e aguardando condições favoráveis à germinação.

A maior densidade de sementes dispersadas (CS) e germinadas (BS) foi observada no Grupo 1 (Tabela 2), o que pode estar associado à maior abertura existente no dossel deste ambiente devido ao histórico de exploração. Este aumento de luminosidade incidente na copa dos indivíduos reprodutivos de Ilex paraguariensis, pode estar favorecendo a frutificação. Segundo Kageyama e Piña-Rodrigues (1993), isso ocorre porque a maior
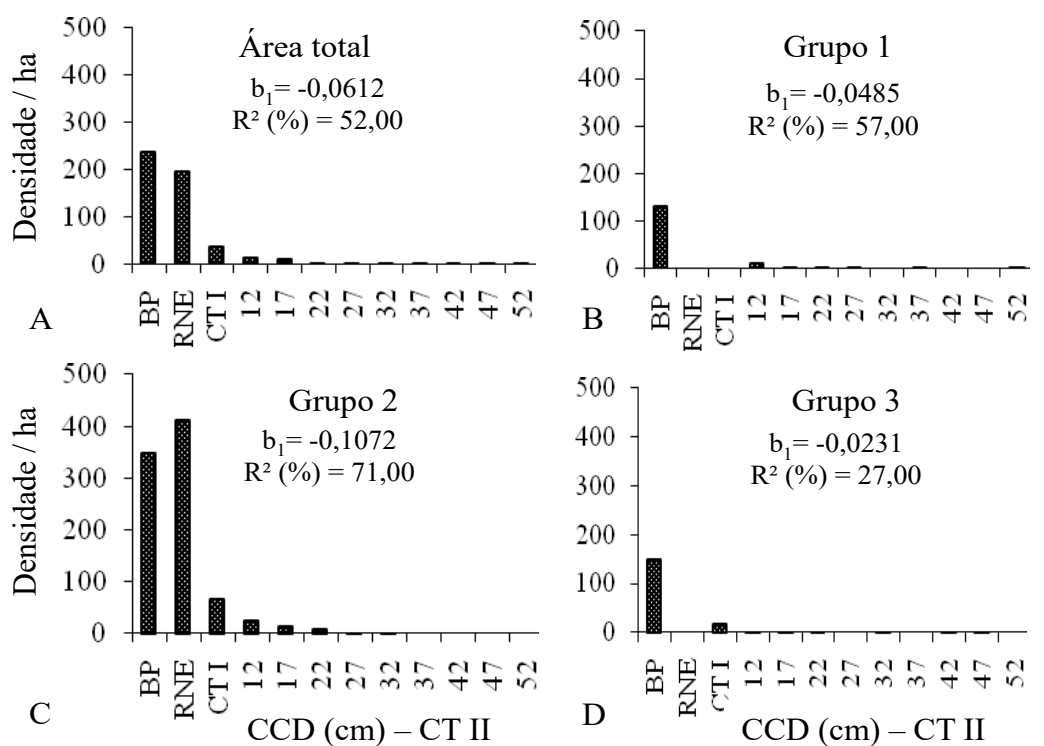

FIGURA 3: Distribuição de frequência para os indivíduos de Ilex paraguariensis em toda a área amostrada (A) e nos Grupos 1 (B), 2 (C) e 3 (D). Os valores referentes à inclinação $\left(b_{1}\right)$ e coeficiente de determinação $\left(\mathrm{R}^{2}\right)$ referem-se ao ajuste de equações para os indivíduos com $\mathrm{DAP} \geq 9,55 \mathrm{~cm}$ (CT II). Centro de classe de diâmetro (CCD).

FIGURE 3: Frequency distribution for individuals of Ilex paraguariensis in all sampled area (A) and Groups 1 (B), 2 (C) and 3 (D). The values for the slope $\left(b_{1}\right)$ and coefficient of determination $\left(R^{2}\right)$ were obtained from adjustment of equations for individuals with $\mathrm{DBH} \geq 9.55 \mathrm{~cm}$ (CT II). Center of diameter class (CCD). 
disponibilidade de luz favorece a iniciação das gemas reprodutivas, propiciando maior florescimento e intensificando a frutificação e entrada de sementes na CS e BS.

O Grupo 2 apresentou as maiores densidades de indivíduos adultos (CT II) e de indivíduos regenerantes de erva-mate (Tabela 2), demonstrando ser uma população com melhor condição de automanutenção na comunidade. Esse equilíbrio entre jovens e adultos pode estar associado ao histórico de menor interferência da área de estudo, que possibilitou condições mais adequadas à regeneração da espécie.

Araucaria angustifolia, Blepharocalyx salicifolius e Ilex paraguariensis apresentaram estruturas populacionais diferenciadas dentro do mesmo ambiente como também nos diferentes ambientes amostrados, muito provavelmente devido às suas distintas características autoecológicas. Considerando as falhas no ciclo regenerativo de algumas populações, a aplicação de intervenções silviculturais planejadas poderia estimular a regeneração das espécies na comunidade, pois as mesmas possuem grande importância ecológica (interações entre flora e fauna) e econômica (produtos madeireiros e não-madeireiros).

Neste contexto, Whitmore (1998) destacou que a silvicultura de florestas nativas tem como objetivo manejar a vegetação para estimular a regeneração de espécies foco, tendo como base ecológica o controle no tamanho das clareiras e sua influência sobre a autoecologia das diferentes espécies. Dessa forma, cada espécie e cada ambiente necessitam ser estudados individualmente, considerando suas respectivas peculiaridades para identificar métodos adequados, que promovam o equilíbrio no ciclo regenerativo das populações.

No caso de Araucaria angustifolia, espécie que predomina na vegetação adulta da Floresta Ombrófila Mista, observou-se que a regeneração natural é possivelmente incapaz de manter a predominância da espécie no ecossistema estudado, exceto para o Grupo 1, no qual a espécie apresentou maior densidade na regeneração. Por conseguinte, considerando que Araucaria angustifolia é espécie heliófila, a sua conservação e o seu manejo poderiam ser favorecidos com a adoção de práticas que visem aumentar a luminosidade no sub-bosque para aumentar a densidade do seu componente regenerativo. Porém, as intervenções devem ser estudadas e planejadas, evitando prejuízos às populações de outras espécies, como Blepharocalyx salicifolius e Ilex paraguariensis.

Nesse sentido, o aumento na incidência de luz pode ser estimulado por tratos como a exclusão gradativa (anelamento) de indivíduos em senescência, podendo-se ainda usufruir da madeira. Contudo, visando minimizar danos na composição florística da comunidade e garantir o aumento do potencial regenerativo das espécies desejáveis, deve-se anelar espécies que apresentem boa densidade e distribuição na área (evitando espécies raras) e aplicar os tratamentos após a dispersão das sementes das espécies desejadas para beneficiar a sua regeneração (CARVALHO, 1992), além de considerar o sistema reprodutivo (plantas monoicas ou dioicas) das espécies desejadas e a importância ecológica de espécies a serem eliminadas. Essas práticas poderiam ser utilizadas em florestas públicas, ou mesmo, pelos proprietários rurais, como estímulo à conservação da Floresta Ombrófila Mista, pois, de acordo com Sanquetta et al. (2000), somente a preservação integral dos fragmentos e a fiscalização não são capazes de garantir este mérito, sendo necessário o estímulo a práticas de manejo em bases sustentáveis, com geração e difusão de tecnologias.

\section{CONCLUSÕES}

Os resultados do estudo permitem concluir que:

a) A estrutura populacional e a regeneração natural não ocorrem de igual forma em todos os ambientes da floresta, sendo necessário considerar este aspecto na conservação e manejo dessas espécies; b) Araucaria angustifolia regenera de forma mais abundante em ambientes que sofreram alteração e que apresentam maior nível de abertura no dossel. A população de Blepharocalyx salicifolius encontra-se em equilíbrio em ambientes menos alterados, sendo influenciada por interferências que modificam a estrutura da vegetação. Ilex paraguariensis tem potencial para permanecer no ambiente por longo prazo, estando presente em todos os mecanismos de regeneração e apresentando maior equilíbrio populacional em ambientes mais conservados; c) A aplicação de intervenções poderia estimular a regeneração das espécies, considerando as falhas no ciclo regenerativo de algumas populações. No entanto, estas práticas devem ser planejadas de acordo com a autoecologia de cada espécie e visando minimizar danos à composição florística. 


\section{AGRADECIMENTOS}

Os autores agradecem ao Professor Doádi Antônio Brena e ao Projeto PELD pelo suporte logístico, bem como a Ezequiel Gasparin, Regis Longhi, Marcelo Krug e Angelo Ebling pelo auxílio na coleta de dados a campo. Agradecemos também Arthur Soligo pelo apoio durante a realização da pesquisa e fornecimento de informações sobre o histórico de interferência na área de estudo, bem como as valiosas sugestões realizadas pelos revisores. Esta pesquisa foi financiada pelo Conselho Nacional de Desenvolvimento Cientifico e Tecnológico-CNPq por meio de bolsa de mestrado concedida ao primeiro autor.

\section{REFERÊNCIAS BIBLIOGRÁFICAS}

ARAUJO, M. M. et al. Caracterização da chuva de sementes, banco de sementes do solo e banco de plântulas em Floresta Estacional Decidual ripária, Cachoeira do Sul, RS, Brasil. Scientia Forestalis, Piracicaba, n. 66, p. 128-141, 2004.

ARAUJO, M. M. et al. Análise de agrupamento em remanescente de Floresta Ombrófila Mista. Ciência Florestal, Santa Maria, v.20, n.1, p.1-18, jan./mar. 2010.

AVILA, A. L. et al. Agrupamentos florísticos na regeneração natural em remanescente de Floresta Ombrófila Mista, RS, Brasil. Scientia Forestalis, Piracicaba, v. 39, n. 91, p. 331-342, set. 2011.

BAZZAZ, F. A. Regeneration of tropical forests: physiological responses of Pioneer and secondary species. In: GÓMEZ-POMPA, A.; WHITMORE, T.C.; HADLEY, M. Rain forest regeneration and management. Paris: The UNESCO Press, 1991, v. 6, p. 91-118.

BEGON, M.; TOWNSEND, C. R.; HARPER, J. L. Ecologia: de indivíduos a ecossistemas. 4 ed. Porto Alegre: Artmed, 2007. 752 p.

BORGHETTI, F. Dormência embrionária. In: FERREIRA, A. G.; BORGHETTI, F. (Orgs.). Germinação: do básico ao aplicado. Porto Alegre: Artmed, 2004. p.109-124.

BRASIL. Instituto Brasileiro do Meio Ambiente e dos Recursos Naturais Renováveis. Floresta Nacional de São Francisco de Paula-RS. Brasília: IBAMA, 2000. 6p.

BRASIL. Lei n. 11.284, de 2 de março de 2006. Gestão de florestas públicas para a produção sustentável; institui, na estrutura do Ministério do Meio Ambiente, o Serviço Florestal Brasileiro - SFB; cria o Fundo Nacional de Desenvolvimento Florestal - FNDF. Citation on computers documents Disponível em: <(http://www.planalto. gov.br/ccivil_03/_Ato2004-2006/2006/Lei/L11284. $\mathrm{htm})>$ Acesso em: 14 de dezembro de 2009.

CALDATO, S. L. et al. Estudo da regeneração natural, banco de sementes e chuva de sementes na reserva genética florestal de Caçador, SC. Ciência Florestal, Santa Maria, v. 6, n. 1, p. 27-38, 1996.

CALDATO, S. L.; LONGHI, S. J.; FLOSS, P. A. Estrutura populacional de Ocotea porosa (Lauraceae) em uma Floresta Ombrófila Mista, em Caçador (SC). Ciência Florestal, Santa Maria, v. 9, n.1, p.89-101, mar./abr. 1999.

CANALEZ, G. G.; CORTE, A. P. D.; SANQUETTA, C. R. Dinâmica da estrutura da comunidade de Lauráceas no período de 1995-2004 em uma Floresta de Araucária no sul do estado do Paraná. Ciência Florestal, Santa Maria, v.16, n.4, p.357-367, 2006.

CARVALHO, J. O. P. Dinâmica de Florestas Naturais e sua implicação para o manejo florestal. In: CURSO DE MANEJO FLORESTAL SUSTENTÁVEL, 1997, Curitiba. Tópicos em manejo florestal sustentável. Colombo: EMBRAPA-CNPF, 1997. p.43-55. (EMBRAPA-CNPF. Documentos, 34).

CARVALHO, J. O. P. Structure and dynamics of a logged over Brazilian Amazonian rain Forest. 1992. 215 f. These (Doctor of Philosophy) - Oxford University, Oxford, 1992.

CARVALHO, P. E. R. Espécies arbóreas brasileiras. Brasília: Embrapa Informações Tecnológicas; Colombo: Embrapa Florestas, 2003. v. $1.1039 \mathrm{p}$.

CARVALHO, P. E. R. Espécies arbóreas brasileiras. Brasília: Embrapa Informações Tecnológicas; Colombo, PR: Embrapa Floresta. 2006. v. 2. 627p.

CHAMI, L. B. et al. Mecanismos de regeneração natural em diferentes ambientes de remanescente de Floresta Ombrófila Mista, São Francisco de Paula, RS. Ciência Rural, Santa Maria, v.41, n.2, p.251-259, 2011.

COSTA JÚNIOR, R. F. et al. Estrutura fitossociológica do componente arbóreo de um fragmento de Floresta Ombrófila Densa na mata sul de Pernambuco, Nordeste do Brasil. Ciência Florestal, Santa Maria, v.18, n.2, p.173-183, abr./jun. 2008.

EMBRAPA. Centro Nacional de Pesquisa de Solos. Sistema brasileiro de classificação de solos. 
Brasília: EMBRAPA - SPI, 1999. 412p.

FINGER, C. A. G. Fundamentos de biometria florestal. Santa Maria: UFSM/CEPEF/FATEC, 1992. 201p.

HARTSHORN, G. S. Neotropical forest dynamics. Tropical Succession, v.12, p.23-30, 1980.

HERMANN, M. L. P.; ROSA, R. O. Relevo. In: IBGE. Geografia do Brasil: Região Sul. Rio de Janeiro: 1990. p.55-111.

HESS, G. et al. Trends in forest composition and size class distribution: implications for wildlife habitat. In: Southern Center for Sustainable Forests. Working Paper. North Carolina: Economic end Ecological Impacts, 2000. 96 p.

JARENKOW, J. A. Composição florística e estrutura da Mata com Araucária na Estação Ecológica de Aracuri, Esmeralda, Rio Grande do Sul. 1985. 86f. Dissertação (Mestrado em Ciências Biológicas) - Universidade Federal do Rio Grande do Sul, Porto Alegre, 1985.

KAGEYAMA, P. Y.; PIÑA-RODRIGUES, F. C. $M$. Fatores que afetam a produção de sementes. In: AGUIAR, I. B. de; PIÑA-RODRIGUES, F. C. M.; FIGLIOLIA, M. D. (Orgs.) Sementes florestais tropicais. Brasília: ABRATES, 1993. p.19-46.

LAMPRECHT, H. Silvicultura nos trópicos: ecossistemas florestais e respectivas espécies arbóreas - possibilidades e métodos de aproveitamento sustentado. Rossdorf: TZ-Ver.Ges., 1990. 343p.

LEITE, P. F. Contribuição ao conhecimento fitoecológico do Sul do Brasil. Ciência \& Ambiente, Santa Maria, v.1, n.1, p.51-73, 2002.

LONGHI, S. J. A estrutura de uma floresta natural de Araucaria angustifólia (Bert.) Ktze, no sul do Brasil. 1980. 198 f. Dissertação (Mestrado em Ciência Florestal) - Universidade Federal do Paraná, Curitiba, 1980.

LONGHI, S. J. et al. Fatores ecológicos determinantes na ocorrência de Araucaria angustifolia e Podocarpus lambertii, na Floresta Ombrófila Mista da FLONA de São Francisco de Paula, RS, Brasil. Ciência Rural, Santa Maria, v.40, n.1, jan./fev. 2010.

LYKKE, A. M. Assessment of species composition change in savanna vegetation by means of woody plants' size class distributions and local information.

Biodiversity and Conservation, Netherlands, v. 7, p.1261-1275, 1998.

MALUF, J.R.T. Nova classificação climática do Estado do Rio Grande do Sul. Revista Brasileira de Agrometeorologia, Santa Maria, v.8, n.1, p.141-150, 2000.

MARTINS, F. R. Estrutura de uma Floresta Mesófila. Campinas: UNICAMP, 1993. 246p. MATTEUCCI, S. D.; COLMA, A. Metodologia para el estudio de la vegetación. Washington: Secretaria General de la Organización de los Estados Americanos - Programa Regional de Desarrollo y Tecnologico, 1982. 169p.

McLAREN, K. P. et al. Predicting species response to disturbance from size class distributions of adults and saplings in a Jamaican tropical dry forest. Plant Ecology, Dordrecht, v.181, p.69-84, 2005.

MOSER, J. M. Solos. In: IBGE. Fundação Instituto Brasileiro de Geografia e Estatística. Geografia do Brasil: Região Sul. Rio de Janeiro, 1990. p.85-111. MWAVU, E. N.; WITKOWSKI, T. F. Population structure and regeneration of multiple-use tree species in a semi-deciduous African tropical rainforest: Implications for primate conservation. Forest Ecology and Management, Amsterdan, v.258, p.840-849, 2009.

NASCIMENTO, A. R. T.; LONGHI, S. J.; BRENA, D. A. Estrutura e padrões de distribuição espacial de espécies arbóreas em uma amostra de Floresta Ombrófila Mista em Nova Prata, RS. Ciência Florestal, Santa Maria, v.11, n.1, p.105-119, mar./abr. 2001.

NORDEN, N. et al. Resilience of tropical rain forests: tree community reassembly in secondary forests. Ecology Letters, v.12, p.385-394, 2009.

NIMER, E. Clima. In: IBGE. Geografia do Brasil: Região Sul. Rio de Janeiro, 1990. p.151-187.

OBIRI, J.; LAWES, M.; MUKOLWE, M. The dynamics and sustainable use of high-value tree species of the coastal Pondoland forests of the Eastern Cape Province, South Africa. Forest Ecology and Management, Amsterdan, v. 66, p.131-148, 2002.

QUADROS, F. L. F. de; PILLAR, V. de P. Transições floresta-campo do Rio Grande do Sul. Ciência \& Ambiente, Santa Maria, v.1, n.1, p.109-118, 2002. REITZ, P. et al. Projeto madeira do Rio Grande do Sul. Itajaí: Herbário Barbosa Rodrigues, 1983. $525 \mathrm{p}$.

RICHARDS, P. W. The tropical rain forest: an ecological study. Cambridge: University Press, 1998. 575p.

RIO GRANDE DO SUL. Secretaria Especial do Meio Ambiente. Inventário florestal contínuo do Rio Grande do Sul. Porto Alegre, 2002. 706 p. (Relatório Técnico).

SABOGAL, C. Regeneration of tropical dry forests 
in Central America, with examples from Nicaragua. Journal of Vegetation Science, Washington, v. 3, p.407-416, 1992.

SANQUETTA, C. R. et al. Dinâmica da composição florística de um fragmento de floresta ombrófila mista no centro-sul do Paraná. Revista Ciências Exatas e Naturais, Irati, n.2, p.77-88, 2000.

SCHNEIDER, P. R.; SCHNEIDER, P. S. P.; SOUZA, C. A. M. Análise de regressão aplicada a Engenharia Florestal. 2.ed. Santa Maria: FACOS, 2009. 294p.

SILVA, J. A. et al. Estrutura e Composição

Florística da Reserva Genética Florestal de Caçador, estado de Santa Catarina. Brasília: Embrapa Recursos Genéticos e Biotecnologia, 2004. 29p. (Boletim de Pesquisa e Desenvolvimento, 68).
SOUZA, A. F. Ecological interpretation of multiple population size structures in trees: The case of Araucaria angustifolia in South America. Austral Ecology, Adelaide, v.32, p.524-533, 2007.

SOUZA, A. F. et al. Regeneration patterns of a longlived dominant conifer and the effects of logging in southern South America. Acta Oecologica, v. 34, p.221-232, 2008.

WHITMORE, T. C. An introduction to tropical rain forests. Oxford: Science Publications, 1998. 282p.

ZANON, M. L. B. Crescimento da Araucaria angustifolia (Bertol.) Kuntze diferenciado por dioicia. 2007. 110f. Tese (Doutorado em Engenharia Florestal) - Universidade Federal de Santa Maria, Santa Maria, 2007. 